\title{
Patients with a Family History of Cancer: Identification and Management
}

\author{
Margaret M. Eberl, MD, MPH, Annette Y. Sunga, MD, MPH, \\ Carolyn D. Farrell, MS, CNP, CGC, and Martin C. Maboney, MD, PhD, FAAFP
}

\begin{abstract}
A family history of certain malignancies, especially breast, ovarian, colorectal, and prostate cancers, can place persons at increased risk of developing these cancers. By constructing a pedigree that includes 3 generations, family physicians can identify patients at increased risk because of family cancer history. Persons at increased cancer risk because of family history warrant a surveillance strategy for early detection. Genetic professionals represent an important resource in assessing genetic risk and possible testing. Persons identified as being at increased risk of various cancers based on their family history should understand the surveillance plan that is recommended and the importance of maintaining a healthy lifestyle and remaining up to date on other cancer screening tests. (J Am Board Fam Pract 2005; 18:211-7.)
\end{abstract}

A family history of cancer is well accepted as an important risk factor for the development of several of the more common cancers. ${ }^{1}$ Approximately 5\% to $10 \%$ of breast, ovarian, and colon cancers are caused by a hereditary cancer syndrome. ${ }^{2,3}$ Advances in molecular genetics have made it possible to identify a small subset of individual patients, and families, who have increased cancer risk because of the presence of cancer susceptibility genes and/or mutations. ${ }^{4}$ In the majority of cases, however, specific mutations have not yet been identified or cancers arise sporadically in the context of various risk factors, including a positive family history. ${ }^{2} \mathrm{Al}-$ though sometimes complicated, the identification of patients with hereditary cancer syndromes, as well as a familial predisposition to cancer, allows for the delivery of appropriate potentially life-saving preventive care. ${ }^{3}$

Whereas screening is used to detect asymptomatic disease among persons deemed to be at average

Submitted, revised, 15 February 2005.

From the Division of Cancer Prevention and Population Sciences (MME, AYS, MCM), Department of Cancer Genetics, Clinical Genetics Service (CDF), Roswell Park Cancer Institute, Buffalo, New York, and Department of Social and Preventive Medicine, School of Public Health and Health Professions (MME, AYS, MCM), Department of Family Medicine, School of Medicine and Biomedical Sciences (MME, AYS, MCM), State University of New York at Buffalo. Address correspondence to Margaret M. Eberl, MD, MPH, Division of Cancer Prevention and Population Sciences, Roswell Park Cancer Institute, Carlton 307, Elm and Carlton Streets, Buffalo, NY 14263 (e-mail: margaret. eberl@roswellpark.org). risk, surveillance refers to strategies for monitoring those at increased risk by virtue of their personal medical history, lifestyle, environmental exposures, and family history. It is estimated that 1 in 50 patients evaluated by a primary care physician is at increased risk because of his or her family history. ${ }^{5}$ Failing to identify these patients may have significant health consequences for the patient and the patient's family. ${ }^{5}$ This article focuses on the identification and management of patients judged to be at increased risk of breast, ovarian, colon, and prostate cancers by virtue of their family history of cancer.

\section{Identifying Patients at Increased Cancer Risk Because of Family History}

Family history is best assessed via construction of a pedigree covering at least 3 generations. In general, family histories of cancer that suggest increased susceptibility include: 2 or more relatives on the same side of the family with the same or a related cancer, earlier than average age of diagnosis (typically $\leq 50$ years of age for most cancers), and the presence of more than 1 primary cancer in a family member, exclusive of metastatic disease. (Although not a focus of this study, the presence of specific medical conditions [eg, ulcerative colitis] may also be associated with an increased risk of developing certain types of cancer.) It is important to note that hereditary cancers also may occur in the less typically affected sex (eg, male breast cancer). ${ }^{4}$ In such cases, evaluation by a genetics professional should 
be considered to be assured of comprehensive assessment and appropriate counseling as a prelude to possible genetic testing. ${ }^{6}$

Although collecting family history data on each family member is essential to identifying patients at increased risk, it is clear that such data are not routinely or comprehensively collected by most physicians. Although most charts contain some information on family history, cancer history, and age at diagnosis, the presence of high-risk lesions (eg, colonic polyps, atypical ductal hyperplasia on breast biopsy) and updated information are often absent. $^{5,7}$

Several barriers to adequate pedigree construction exist. Time constraints on physicians may make pedigree construction challenging; furthermore, physicians may feel unprepared to discuss genetic issues and manage the emotions that may arise. ${ }^{5}$ Computerized tools and preintake questionnaires may help physicians overcome some of these barriers. ${ }^{5}$ Physician concerns regarding potential medicolegal issues and employer discrimination may also hinder efforts to screen patients for familial cancer risk. ${ }^{3}$ Ultimately, physicians will need to view genetic counseling as an ongoing communication process. ${ }^{8}$ National guidelines, and other resources, can assist primary care physicians in discussing genetic risk with their patients.

The American Society of Clinical Oncology (ASCO) and the National Comprehensive Cancer Network (NCCN), as well as other professional specialty societies, have developed recommendations regarding genetic counseling and possible cancer gene testing for patients with a personal and/or family history of cancer that meets certain syndrome-specific criteria based on clinical, pathologic, and/or testing features (Strength of Recommendation Taxonomy [SORT] level C). ${ }^{9,10}$ ASCO emphasizes that all genetic testing must be accompanied by comprehensive pre-test and post-test counseling, as well as informed consent. ${ }^{9}$ The full set of NCCN guidelines can be accessed at http:// www.nccn.org. ASCO clinical practice guidelines can be accessed at http://www.asco.org/ac.

\section{Family History and Breast/Ovarian Cancer}

A family history of breast cancer and/or ovarian cancer increases risk for both of these cancers. Women with at least one first-degree relative with breast cancer have 2 to 4 times the risk of develop- ing breast cancer. ${ }^{11}$ Factors such as a greater number of affected relatives, younger age at diagnosis, and closer kinship each further increases the risk. $^{12,13}$

Approximately $5 \%$ to $10 \%$ of breast cancer cases are caused by known mutations in cancer susceptibility genes, the most common being BRCA1 (BReast CAncer 1) and BRCA2, identified in the early to mid-1990s. ${ }^{14,15}$ These mutations are inherited in an autosomal-dominant manner. ${ }^{16}$ Although the prevalence of BRCA1 mutations in the general population is estimated to be 1 in $800,{ }^{17}$ the prevalence of BRCA2 is unknown. ${ }^{18}$ Whereas hundreds of different mutations in these genes have been identified across affected families, ${ }^{19} 3$ specific mutations are more common $(2.5 \%)$ in women of Ashkenazi Jewish ancestry. ${ }^{20,21}$ An extensive study of Jewish BRCA mutation carriers reported the lifetime risk of breast cancer was approximately $56 \%$ (95\% CI, $40 \%$ to $73 \%$ ), whereas the risk for ovarian cancer was $16 \%$ (95\% CI, $6 \%$ to $28 \%) .{ }^{15,20}$ Other reports have estimated that by age $60,54 \%$ (95\% CI, $27 \%$ to $71 \%$ ) of BRCA1 mutation carriers may have breast cancer, whereas 30\% (95\% CI, $8 \%$ to $47 \%$ ) may have ovarian cancer. ${ }^{9}, 11$ Genetic specialists use various risk assessment models to estimate risk for breast cancer and the likelihood of detecting a BRCA mutation, as well as providing clinical and pedigree evaluation to assess for this and other breast cancer genetic syndromes. ${ }^{22}$

Identification and surveillance strategies for women who are at increased risk for breast cancer are summarized in Table 1. Patients with an increased risk of breast cancer because of family history factors should be informed of this concern and instructed regarding a surveillance plan. Appropriate surveillance is dependent on mammographic screening beginning either at 30 years of age or 10 years before the earliest age at breast cancer diagnosis in a family member (SORT level C). ${ }^{23}$ It has been found that BRCA mutation carriers may benefit from mammography beginning at age 25 years or sooner, depending on the earliest diagnosis in the family. ${ }^{24}$ So far, the utility of breast magnetic resonance imaging for breast cancer surveillance in high-risk women has not been established. Although evidence is inconclusive, women at increased risk for breast cancer may benefit from lifestyle changes, including increased exercise, as well as chemoprevention with agents such as tamoxifen. ${ }^{3}$ 
Table 1. Identification and Management for Patients at Increased Risk of Breast Cancer based on Family Cancer History

Identification of persons at 'increased risk' because of family cancer history

Breast cancer risk increased among:

Women with a mother and/or sister(s), daughter(s) with breast cancer, especially if diagnosed before age 50 years.

Women who have a father and/or paternal relatives (grandmother or aunts) with breast cancer, especially if diagnosed before age 50 years.

Women who have maternal relatives (grandmother or aunts) with breast cancer, especially if diagnosed before age 50 years.

Families with a history of breast and/or ovary and/or colon and rectum cancers among mothers/fathers, brothers/sisters, children, and/or maternal or paternal relatives.

Families with a history of cancers and/or inherited conditions or known/suspected gene mutation associated with breast cancer risk (eg, BRCA1/2 mutations, Li Fraumeni syndrome, Ashkenazi Jewish ancestry).

Surveillance recommendations for persons at 'increased risk' because of family cancer history

Beginning at age 30 years (or 10 years before age of earliest case in family):

Evaluate family history of cancer and overall health status of patient.

Discuss risk status and overall health status with patient and plans for ongoing surveillance, including age to begin mammography.

Consider whether involvement in a prevention trial is an option.

Consider a consultation with a genetic counselor for risk assessment and possible genetic testing.

Surveillance recommendations (Table 2) for women at increased risk of ovarian cancer based on family history include pelvic examination, CA-125 testing, and vaginal ultrasound monitoring (SORT level C). ${ }^{24,25}$ Frequency of testing is best addressed via referral to a comprehensive cancer center where broad clinical experience and a variety of support services are also available.

Ideally, women with a family history of breast and/or ovarian cancer can be referred for genetic counseling and, if judged appropriate, offered genetic testing (SORT level C). ${ }^{26}$ Testing often begins with an affected relative, if available. If a mutation is identified in an affected person, genetic testing for the specific mutation can be extended to other family members. ${ }^{4}$ Based on an autosomaldominant pattern of inheritance, the children of a carrier will have a $50 \%$ chance of having the same mutation. ${ }^{6}$ Prophylactic mastectomy for mutation
Table 2. Identification and Management for Patients at Increased Risk of Ovarian Cancer Based on Family Cancer History

Identification of persons at 'increased risk' because of family cancer history

Ovarian cancer risk increased among:

Women who have a mother and/or sister(s), daughter(s), or grandparent(s) with breast cancers and/or ovarian cancers, especially if one or more is diagnosed before age 50 years.

Women with a personal or family history of breast, endometrial, or colorectal cancer.

Surveillance recommendations for persons at 'increased risk' because of family cancer history

All ages:

Evaluate comprehensive family history of cancer and overall health status of patient.

Discuss risk status, and overall health status with patient, and plans for ongoing surveillance including possible referral to specialist, ultrasound monitoring, pelvic examination and CA-125 testing.

Consider whether involvement in a prevention trial is an option.

Consider a consultation with a genetic counselor for risk assessment and possible genetic testing.

carriers has been shown to reduce the risk of breast cancer by as much as $90 \%$, as well as increase life expectancy by as much as 5 years. ${ }^{3}$ Similarly, prophylactic oophorectomy may decrease subsequent breast and ovarian cancer rates and increase life expectancy. ${ }^{3}$ For both these procedures, however, any potential benefits must be weighed along with long-term quality-of-life issues.

Finally, it should be noted that application of empiric risk breast cancer assessment models (eg, Gail or Claus) should not automatically occur for women at increased risk because of family history, because these models have limitations yielding both underestimates and overestimates of risk. ${ }^{3}$ In general, Gail risk estimate figures are typically higher than Claus risk estimates, with both models being more accurate for women at low to moderate risk. The use of mutation probability models should be used in those patients whose family history is suggestive of the presence of a genetic mutation. ${ }^{3}$

\section{Family History and Colorectal Cancer}

Risk of colorectal cancer is increased among persons with one or more affected first-degree family members ${ }^{27}$; the relative risk exceeds 5 among persons with an affected first-degree relative diagnosed before age 45 years. ${ }^{27}$ In families with more than one person diagnosed with colorectal cancer, the 
cause may be multifactorial, including environmental influences and inheritance of genetic factors. ${ }^{28}$ Cases of colorectal cancer (CRC) can be classified into 3 categories: (1) sporadic (no family history), accounting for approximately $60 \%$ of all cases; (2) familial (several affected family members), accounting for $30 \%$ of cases; and (3) hereditary (caused by defined genetic syndromes), which represents the remainder. ${ }^{29}$ Colorectal cancer screening guidelines issued by the American Cancer Society classify these categories as "average," "moderate," and "high risk," respectively. ${ }^{29}$

Colorectal cancers in a family can be caused by mutations in a single gene. Familial adenomatous polyposis (FAP) is an autosomal-dominant multisystem disorder, with the classic form characterized by the formation at an early age of hundreds to thousands of adenomatous polyps within the colon and rectum. ${ }^{30}$ Although FAP accounts for $1 \%$ of all colorectal cancer cases, persons with FAP have a nearly $100 \%$ chance of developing cancer by age $50 .{ }^{30}$ Because FAP is caused by mutations in the adenomatous polyposis coli $(A P C)$ gene, genetic testing can be offered to at-risk patients and their families (SORT level C). ${ }^{30}$ Affected persons, and others deemed to be at risk for FAP, require surveillance beginning as early as age 10 to 15 years (SORT level C). ${ }^{31}$

Hereditary nonpolyposis colorectal cancer (HNPCC) is another autosomal-dominant condition manifesting as early-onset CRC, as well as other malignancies, involving the endometrium, small bowel, ureter, or renal pelvis. ${ }^{28}$ HNPCC accounts for $6 \%$ of all colorectal cancers and is not typically preceded by formation of polyps. ${ }^{32}$ Persons with HNPCC have a lifetime risk of CRC ranging between $70 \%$ and $80 \% .{ }^{32,33}$ Women with HNPCC have a lifetime risk of $30 \%$ to $60 \%$ for developing endometrial cancer. ${ }^{34}$

In 1990, the Amsterdam criteria were developed by the International Collaborative Group to aid in the identification of HNPCC families. The classic guidelines recommend further investigation of those patients for whom CRC occurs in 3 or more family members, affecting 2 successive generations, involving at least 2 persons who are first-degree relatives of another, and include one case diagnosed before age 50 years. Furthermore, FAP should be excluded in all cases of CRC, and tumors must be verified by pathologic evaluation. ${ }^{35}$ The Amsterdam criteria were expanded in 1999 to include
Table 3. Identification and Management for Patients at Increased Risk of Colorectal Cancer Based on Family Cancer History

Identification of persons at 'increased risk' because of family cancer history

Colorectal cancer risk increased among:

Persons who have an FDR with cancer of the colon or rectum.

Persons who have a maternal or paternal relative (aunt/ uncle, grandparent) with colorectal cancer, especially if diagnosed before age 50 years.

Persons with a personal and/or family history of inherited conditions associated with colon cancer (eg, HNPCC and FAP).

Families affected by cancers of the uterus, breast, and/ or ovary among first-degree or second-degree relatives (consider consultation with a genetic counselor/specialist).

Surveillance recommendations for persons at 'increased risk'

because of family cancer history

Beginning at age 40 years (or 10 years before age of earliest case in family):

Evaluate comprehensive family history of cancer and overall health status of patient.

Discuss risk status, and overall health status with patient, and plans for ongoing surveillance including age at which to begin colonoscopy and plan for ongoing surveillance.

Persons who have a mother, father, or sibling who has been diagnosed with colorectal cancer, require a colonoscopy at age 40 years, or earlier, depending upon age of onset in the family.

Persons at increased risk of colorectal cancer require colonoscopy; no other test may substitute.

Consider a consultation with a genetic counselor for risk assessment, and possible genetic testing.

Consider whether involvement in a prevention trial is an option.

HNPCC, hereditary nonpolyposis colorectal cancer; FAP, familial adenomatous polyposis; FDR, first-degree relative.

other HNPCC-associated tumors (eg, cancers of the endometrium, small bowel, ureter, or renal pelvis). ${ }^{36}$ For the HNPCC-affected family, surveillance guidelines from the NCCN recommend colonoscopy beginning at age 20 to 25 years, or 10 years before the youngest age at diagnosis in the families. Colonoscopy should be repeated every 1 to 2 years until age 40 years, when surveillance colonoscopy should be performed annually (SORT level C). Among affected women, transvaginal ultrasound or endometrial biopsy can be considered beginning at age 25 (SORT level C). ${ }^{31}$

Table 3 summarizes the identification and management of persons at increased risk for colorectal cancer because of family history. Beginning at age 40 years (or 10 years before the age of the earliest 
onset in the family), persons with a mother, father, or sibling who has been diagnosed with colorectal cancer require a colonoscopy (SORT level C). ${ }^{31}$ The interval to the next colonoscopy is generally determined after the initial evaluation and may range from 1 to 5 years. Persons undergoing surveillance for increased risk of colorectal cancer require colonoscopic examination; no other test can be substituted. ${ }^{31}$

\section{Family History and Prostate Cancer}

Men with a brother or father affected by prostate cancer have approximately twice the risk of developing prostate cancer themselves; risk increases as the number of affected family members increases (SORT level B). ${ }^{37}$ In addition, men from families with a history of breast and/or ovarian cancers may also be at increased risk for prostate cancer; consultation with a genetics professional for risk assessment and possible testing for BRCA-1 and BRCA-2 mutations should be considered. ${ }^{6,38}$

Both the ACS and NCCN recommend surveillance via digital rectal examination and prostatespecific antigen testing beginning at age 45 years among men with 2 or more affected first-degree relatives (SORT level C). ${ }^{23,39}$ The American Urologic Association (AUA) recommends surveillance starting at age 40 years for men with 1 affected first-degree relative (SORT level C). ${ }^{40}$

As presented in Table 4, surveillance for prostate cancer should begin at age 40 years (or 10 years before the age of earliest diagnosis in an affected family member) and include annual digital rectal examination and prostate-specific antigen testing (SORT level C). ${ }^{41}$ Similar to issues relating to prostate cancer screening, there is insufficient evidence that prostate cancer surveillance among patients at increased risk results in reduced morbidity or mortality or improved survival. Regardless of when surveillance is begun, all patients should be counseled regarding the risks, benefits, and uncertainties of various modalities for prostate cancer surveillance. $^{39}$

\section{Counseling High-Risk Patients}

Patients are increasingly becoming aware of the need to discuss family medical history with their physicians. It has been reported that patient inquiry represents the primary motivator for physicians ordering genetic tests. ${ }^{3}$ Although evidence support-
Table 4. Identification and Management for Patients at Increased Risk of Prostate Cancer Based on Family Cancer History

Identification of persons at 'increased risk' because of family cancer history

Prostate cancer risk increased among:

Men who have a father, brother, or son with cancer of the prostate.

Men with a mother or sister who has been diagnosed with ovarian cancer.

Families with a history of breast and/or ovarian cancer(s) among first-degree or second-degree relatives may be at increased risk (consider consultation with a genetic counselor/specialist).

Surveillance recommendations for persons at 'increased risk' because of family cancer history

Beginning at age 40 years (or 10 years before age of earliest case in family):

Evaluate comprehensive family history of cancer and overall health status of patient.

Discuss risk status and overall health status with patient and plans for ongoing surveillance, including possible PSA testing starting at age 40 years, or earlier, depending upon age of onset in the family (be certain to discuss the risks and benefits of PSA testing and plan for ongoing surveillance).

Consider a consultation with a genetic counselor for risk assessment.

Consider whether participation in a prevention trial is an option.

PSA, prostate-specific antigen.

ing a clear benefit of counseling may be unavailable, it is clear that patients will continue to look to their family physicians for guidance in this area.

Persons identified as being at increased risk for various cancers based on their family history should have an opportunity to have questions answered either by their family physicians or through referral to a genetics professional. These patients should understand the surveillance plan that is recommended, as well as the importance of remaining up to date on other cancer screening tests. All patients should be counseled on the benefits of a tobaccofree lifestyle, a diet rich in fruits and vegetables, maintenance of a healthy body weight, and participation in regular exercise. Opportunities to participate in prevention and early detection trials are available through comprehensive cancer centers and academic health centers.

\section{Conclusion}

As our understanding of the genetic components of cancer continue to rapidly expand, several barriers to identifying and counseling at-risk families re- 
main. ${ }^{4}$ Current genetic testing techniques are less than $100 \%$ sensitive and specific, leaving negative results open to misinterpretation. ${ }^{1,6}$ In addition, genetic tests are only useful for identifying known mutations. Such limitations highlight the importance of appropriate pre-test and post-test genetic counseling, current knowledge of relevant genetic information and resources, and commitment to the process of informed consent for patients interested in genetic testing. ${ }^{6}$ Furthermore, genetic testing results are relevant to other family members, because this information can have an impact on their health care and medical decisions and often raises concerns regarding health and life insurance and employment and reproductive issues. ${ }^{6}$ In these situations, consultation with a genetics professional can be helpful. ${ }^{6}$

Identifying patients at increased cancer risk because of family cancer history and/or hereditary cancer syndromes begins by obtaining an accurate detailed family history to determine cancer risk. Family history is best assessed via construction of a pedigree that includes at least 3 generations. Even after preparation of a comprehensive pedigree, uncertainties can remain. Clinicians must be able to assess available information and discuss the risks and benefits of genetic testing with their patients. Family physicians are well positioned to use their skills in identifying patients at increased cancer risk because of family history and/or genetic syndromes and to encourage compliance with surveillance strategies.

\section{References}

1. Offit K, Brown K. Quantitating familial cancer risk: a resource for clinical oncologists. J Clin Oncol 1994;12:1724-36.

2. Chew HK. Genetic evaluation of cancer: the importance of family history. Tex Med 2001;97:40-5.

3. Sifri R, Gangadharappa S, Acheson LS. Identifying and testing for hereditary susceptibility to common cancers. CA Cancer J Clin 2004;54:309-26.

4. Weitzel JN. Genetic cancer risk assessment. Putting it all together. Cancer 1999;86(11 Suppl):2483-92.

5. Murff HJ, Byrne D, Syngal S. Cancer risk assessment: quality and impact of the family history interview. Am J Prev Med 2004;27:239-45.

6. Ang P, Garber JE. Genetic susceptibility for breast cancer: risk assessment and counseling. Semin Oncol 2001;28:419-33.

7. Sifri RD, Wender R, Paynter N. Cancer risk assessment from family history: gaps in primary care practice. J Fam Pract 2002;51:856.
8. Daly M, Farmer J, Harrop-Stein C, et al. Exploring family relationships in cancer risk counseling using the genogram. Cancer Epidemiol Biomarkers Prev 1999;8:393-8.

9. Statement of the American Society of Clinical Oncology: genetic testing for cancer susceptibility, Adopted on February 20, 1996. J Clin Oncol 1996; 14:1730-6; discussion 1737-40.

10. National Comprehensive Cancer Network. Clinical practice guidelines in oncology [monograph on the Internet]. Jenkintown (PA): National Comprehensive Cancer Network; 2005 [cited 2005 Jan 18]. Available from: http://www.nccn.org/professionals/ physician_gls/default.asp.

11. Pharoah PD, Day NE, Duffy S, Easton DF, Ponder BA. Family history and the risk of breast cancer: a systematic review and meta-analysis. Int $\mathrm{J}$ Cancer 1997;71:800-9.

12. Colditz GA, Willett WC, Hunter DJ, et al. Family history, age, and risk of breast cancer. Prospective data from the Nurses' Health Study. JAMA 1993; 270:338-43.

13. Slattery ML, Kerber RA. A comprehensive evaluation of family history and breast cancer risk. The Utah Population Database. JAMA 1993;270:1563-8.

14. Miki Y, Swensen J, Shattuck-Eidens D, et al. A strong candidate for the breast and ovarian cancer susceptibility gene BRCA1. Science 1994;266:66-71.

15. Wooster R, Bignell G, Lancaster J, et al. Identification of the breast cancer susceptibility gene BRCA2. Nature 1995;378:789-92.

16. Claus EB, Schildkraut JM, Thompson WD, Risch NJ. The genetic attributable risk of breast and ovarian cancer. Cancer 1996;77:2318-24.

17. Ford D, Easton DF, Peto J. Estimates of the gene frequency of BRCA1 and its contribution to breast and ovarian cancer incidence. Am J Hum Genet 1995;57:1457-62.

18. Emery J, Lucassen A, Murphy M. Common hereditary cancers and implications for primary care. Lancet 2001;358:56-63.

19. Myriad Genetics [homepage on the Internet]. Salt Lake City (UT): Myriad Genetics [cited 2005 Feb 7]. Available from: http://www.myriad.com/index.htm.

20. Struewing JP, Hartge $P, W$ acholder $S$, et al. The risk of cancer associated with specific mutations of BRCA1 and BRCA2 among Ashkenazi Jews. N Engl J Med 1997;336:1401-8.

21. Lynch HT, Snyder CL, Lynch JF, Riley BD, Rubinstein WS. Hereditary breast-ovarian cancer at the bedside: role of the medical oncologist. J Clin Oncol 2003;21:740-53.

22. Evans DG, Lalloo F. Risk assessment and management of high risk familial breast cancer. J Med Genet 2002;39:865-71.

23. Smith RA, Saslow D, Sawyer KA, et al. American Cancer Society guidelines for breast cancer screening: update 2003. CA Cancer J Clin 2003;53:141-69. 
24. Daly M, Bryant E, Buys S, Ellis, CL. NCCN Practice Guidelines: genetic/familial high-risk assessment: breast and ovarian [monograph on the Internet]. Jenkintown (PA): National Comprehensive Cancer Network; 2004 [cited 2005 Jan 18]. Available from: http://www.nccn.org/professionals/physician_ gls/PDF/genetics_screening.pdf.

25. Lewis S, Menon U. Screening for ovarian cancer. Expert Rev Anticancer Ther 2003;3:55-62.

26. Hoskins KF, Stopfer JE, Calzone KA, et al. Assessment and counseling for women with a family history of breast cancer. A guide for clinicians. JAMA 1995;273:577-85.

27. Fuchs CS, Giovannucci EL, Colditz GA, Hunter DJ, Speizer FE, Willett WC. A prospective study of family history and the risk of colorectal cancer. N Engl J Med 1994;331:1669-74.

28. Ivanovich JL, Read TE, Ciske DJ, Kodner IJ, Whelan AJ. A practical approach to familial and hereditary colorectal cancer. Am J Med 1999;107: $68-77$.

29. Smith RA, von Eschenbach AC, Wender R, et al. American Cancer Society guidelines for the early detection of cancer: update of early detection guidelines for prostate, colorectal, and endometrial cancers. Also: update 2001-testing for early lung cancer detection. CA Cancer J Clin 2001;51:38-75; quiz 77-80.

30. Burt RW. Familial risk and colon cancer. Int J Cancer 1996;69:44-6.

31. Levin B, Barthel J, David D, et al. Clinical practice guidelines in oncology, colorectal screening, version 1 [monograph on the Internet]. Jenkintown (PA): National Comprehensive Cancer Network; 2005 [cited 2005 Jan 18]. Available from: http://www.nccn.org/ professionals/physician_gls/PDF/colorectal_screening. pdf.

32. Lynch HT, Smyrk T, Lynch JF. Overview of natural history, pathology, molecular genetics and manage- ment of HNPCC (Lynch syndrome). Int J Cancer 1996;69:38-43.

33. Lynch HT, Smyrk T, Lynch J. An update of HNPCC (Lynch syndrome). Cancer Genet Cytogenet 1997;93:84-99.

34. Dunlop MG, Farrington SM, Carothers AD, et al. Cancer risk associated with germline DNA mismatch repair gene mutations. Hum Mol Genet 1997; 6:105-10.

35. Vasen HF, Mecklin JP, Khan PM, Lynch HT. The International Collaborative Group on HNPCC. Dis Colon Rectum 1991;34:424-5.

36. Vasen HF, Watson P, Mecklin JP, Lynch HT. New clinical criteria for hereditary nonpolyposis colorectal cancer (HNPCC, Lynch syndrome) proposed by the International Collaborative group on HNPCC. Gastroenterology 1999;116:1453-6.

37. Steinberg GD, Carter BS, Beaty TH, Childs B, Walsh PC. Family history and the risk of prostate cancer. Prostate 1990;17:337-47.

38. Ford D, Easton DF, Bishop DT, Narod SA, Goldgar DE. Risks of cancer in BRCA1-mutation carriers. Breast Cancer Linkage Consortium. Lancet 1994; 343:692-5.

39. Scardino P, Bahnson R, Hanks G, Trump D, et al. Clinical practice guidelines in oncology. Prostate cancer. Version 1 [monograph on the Internet] Jenkintown (PA): National Comprehensive Cancer Network; 2003 [cited 2005 Jan 18]. Available from: http://www. nccn.org/professionals/physician_gls/PDF/prostate. pdf.

40. Prostate-specific antigen (PSA) best practice policy. American Urological Association (AUA). Oncology (Huntingt) 2000;14:267-72, 277-8, 280 passim.

41. American Urological Association [homepage on the Internet]. Linthicum (MD): American Urological Association; c2005 [cited 2005 Jan 18]. Available from: http://www.urologyhealth.org/index.cfm. 